\title{
DGEN Aeropropulsion Research Turbofan (DART): Lossless Projection of Measured Engine Noise Spectra to a 1-Foot-Radius Arc
}

\author{
Cliff Brown* and Dan Sutliff ${ }^{\dagger}$ \\ NASA Glenn Research Center, Cleveland, OH, 44135, USA
}

\begin{abstract}
Baseline noise and aerodynamic data have been acquired for the DGEN Aeropropulsion Research Turbofan (DART) test rig. The DART is a fully-mobile engine test rig featuring a DGEN380 geared turbofan producing approximately $500 \mathrm{lbs}$. of thrust at sea level and a self-contained control room. Baseline noise data were acquired using 5 microphone arrays, varying distance, configuration, and angle to reflect the measurement locations at several other test facilities. Noise data were acquired at one array location on each test day to establish the repeatability of the measurements. The noise data from the different arrays is analyzed to show the limitations of projecting the results to a common radius when the noise sources are distributed and the measurement location is not in the geometric far-field.
\end{abstract}

\section{Nomenclature}

$10 F T \quad 30$-microphone 10 -foot radius arc array

$88 S L \quad 30$-microphone 88-inch sideline array

$\phi \quad$ observation angle relative to the zenith (azimuthal or roll angle)

$\theta \quad$ observation angle relative to the upstream jet axis (polar or yaw angle)

ES $\quad$ engine speed as \% of RPMc

$f \quad$ frequency $(\mathrm{Hz})$

$24 \mathrm{FF} \quad$ overhead 24-microphone array in the AAPL

$\mathrm{BPF} \quad$ Blade passing frequency

C Compressor blade passing tone

F $\quad$ Fan blade passing tone

HPT High-pressure turbine

LPT Low-pressure turbine

RPMc fan speed corrected to standard atmospheric conditions

\section{Introduction}

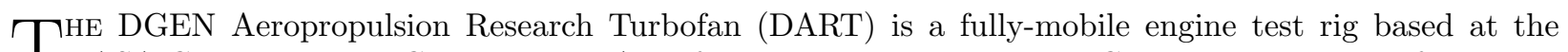
1 NASA Glenn Research Center. The DART features a Price Induction DGEN380 geared turbofan engine connected to a truck-based control room. The DGEN380 is a dual-spool 7.6 bypass-ratio separate-flow geared turbofan engine designed for 4-5 seat twin-engine general aviation class aircraft. The DGEN380 is capable of producing approximately $500 \mathrm{lbs}$. of thrust at sea level. As a test bed for engine research, the DGEN380 can be modified to study how various aspects of engine design impact noise and performance. After and initial assessment in $2014,{ }^{1}$ the first test entry recorded baseline noise data and investigated the effect of a three-degree-of-freedom acoustic inlet liner to reduce forward-radiated fan noise. ${ }^{2}$

The DART test rig is designed to be fully-mobile. The engine, truck-based control room, and supporting hardware can all be stowed, moved, and redeployed in wind tunnel, semi-indoor, or outdoor test facilities.

*Research Engineer, Acoustics Branch, 21000 Brookpark Rd., AIAA Member.

${ }^{\dagger}$ Research Engineer, Acoustics Branch, 21000 Brookpark Rd., AIAA Member 
Therefore, the acoustic baseline test uses arc and sideline microphone arrays, located at different distances on both sides of the engine, to acquire reference acoustic data at locations expected for future tests in the Aero-Acoustic Propulsion Laboratory (AAPL) and the 9x15 Low-Speed Wind Tunnel (9x15 LSWT). These data serve two purposes: (1) to aid in planning future tests by identifying advantages and disadvantages of different microphone arrays, with particular emphasis on data scaling between microphone arrays, and (2) to serve as a quality control database when the DART is first tested in a different facility.

Each microphone array design has advantages and disadvantages that depend on the purpose of the test and any spatial restrictions at the test facility. An arc array placed close to the inlet, for example, will be better at isolating fan noise sources than one placed far away (which will be better at measuring the total engine noise from inlet and exhaust sources). Sideline arrays are more compact in the radial direction so they fit into wind tunnels where arc arrays are impractical but must cover a much longer axial distance to measure the far upstream and downstream angles. Two arc arrays, one focused on the inlet and exhaust and one far away to measure the total engine noise, and a sideline array, matching the traversing microphone in the 9x15 LSWT, were used during the DART baseline test. Combined, this noise database provides insight into the effect of measurement distance and can be used to establish expectations when first testing in a new facility or with a new microphone array in an existing facility.

This paper (1) documents the repeatability of noise measurements using the DART and (2) investigates the choice of microphone array location, with particular focus on projecting the data to a common radius. These data and analyses can be used for future test planning and as validation for noise prediction tools or methods.

\section{Experimental Setup and Data Acquisition}

\section{II.A. Test Facility}

The DART acoustic baseline test was run in the Aero-Acoustic Propulsion Laboratory (AAPL) at the NASA Glenn Research Center (GRC). The AAPL is a geodesic dome (65-foot radius) lined with sound absorbing wedges to create an anechoic environment for frequencies above $200 \mathrm{~Hz}$. The DGEN380 was mounted in the large acoustic arena normally used for the Nozzle Acoustic Test Rig (NATR) allowing use of the NATR overhead microphone array $(24 \mathrm{FF})$ as well as sufficient space for several pole-mounted arc and sideline arrays (Figure 1). The floor and nearby test jet rigs (NATR,SHJAR) were covered with sound absorbing wedges for all DART acoustic test runs. The DART fuel system and control room truck were located outside the AAPL as a safety precaution and to reduce the background noise inside the AAPL. An inlet control device (ICD) was attached to the DGEN380 to provide smooth flow to the engine (Figure 2). Details on the AAPL can be found in [3].

The AAPL uses a grid coordinate system to locate test articles and measurement sensors relative to the center of the dome. This systems allows for easier and more accurate test setup. The array center locations used for DART baseline test, defined in the AAPL coordinate system, are given in Table 1. The flight axis direction vector is also shown in Table 1 . The flight axis direction vector to identify a rotation of the model coordinate system relative to the AAPL grid system; in this case, the DGEN flight axis aligns directly with the AAPL grid so no rotation is required (as described in Section II.C). The transformed coordinate system, referenced to the DGEN, is shown in Figure 2.

Table 1: Coordinates of the DGEN inlet plane, center point fan exhaust plane, and core exhaust plane in the AAPL grid system. Note that the engine center is defined as the center point between the inlet and fan exhaust plane and $z=0$ is defined 10 -feet above the dome floor.

\begin{tabular}{|c|c|c|c|}
\hline Location & $\mathrm{x}(\mathrm{ft})$ & $\mathrm{y}(\mathrm{ft})$ & $\mathrm{z}(\mathrm{ft})$ \\
\hline DGEN Inlet & -3.13 & 24.18 & -4.79 \\
\hline DGEN Center & -1.58 & 24.19 & -4.79 \\
\hline DGEN Fan Exhaust & -0.04 & 24.21 & -4.79 \\
\hline DGEN Core Exhaust & 1.46 & 24.23 & -4.79 \\
\hline Flight Axis & 1.0 & 0.0 & 0.0 \\
\hline
\end{tabular}




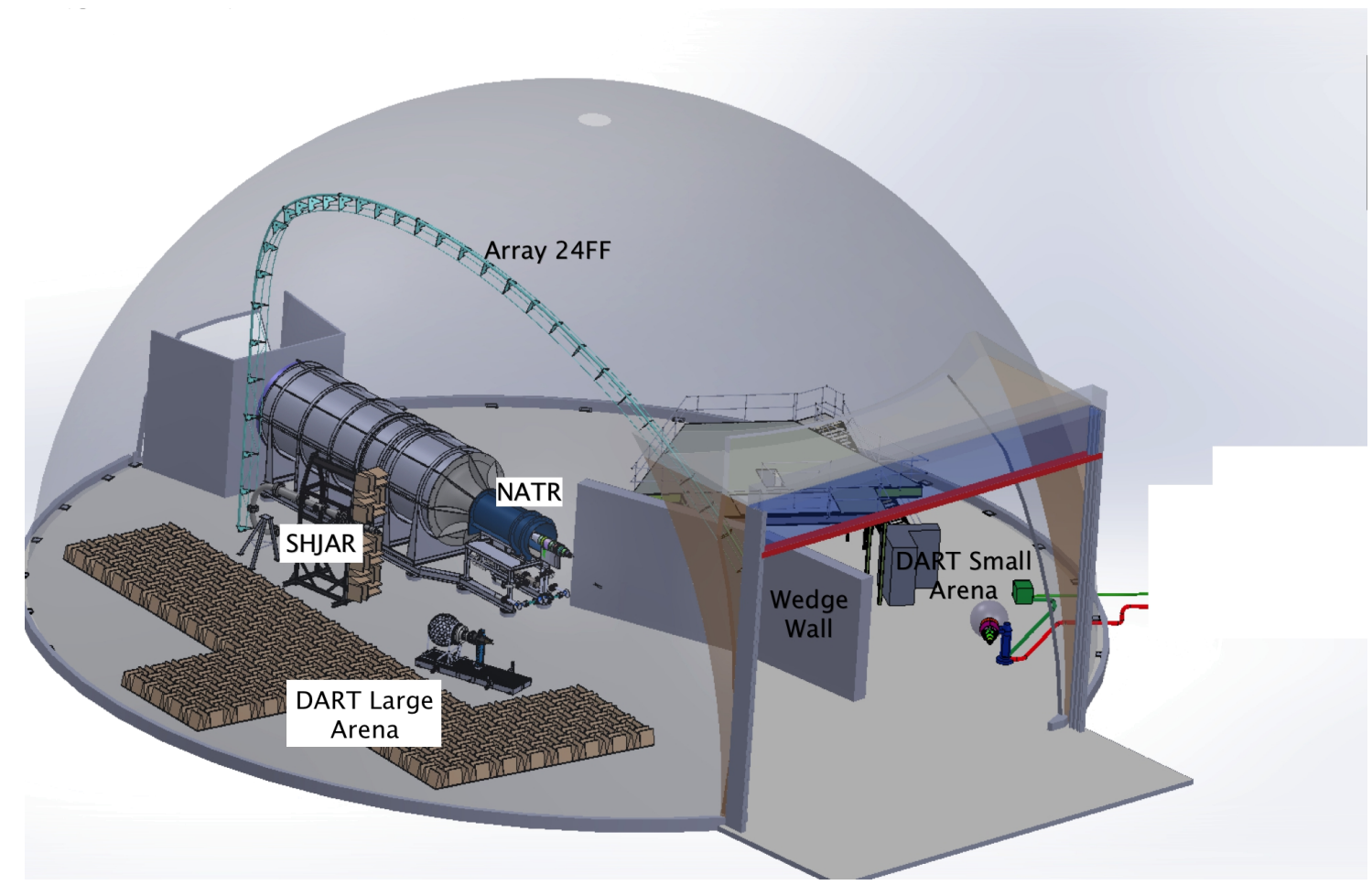

Figure 1: Location of the DGEN380 small and large acoustics test arenas in the AAPL. Note that the 24FF microphone array is an arc centered on the NATR (which would be covered in acoustic wedges for DART testing).

\section{II.B. Microphone Arrays}

Three microphone arrays were used to acquire the baseline acoustic signature of the DGEN380 engine: a 10 -foot radius arc (10FT) array, an 88-inch sideline (88SL) array, and the AAPL overhead (24FF) array. The 10FT and 88SL arrays were used on both sides of the DGEN380 to measure the effect of the engine support pylon on the sound field. ${ }^{4}$

\section{II.B.1. 10-Foot Arc Array (10FT)}

The 10-foot arc array (10FT) consists of 30 microphones on two arcs: 15 centered on the engine inlet plane and 15 centered on the engine exhaust plane of the bypass duct. These arrays are intended to focus the inlet and exhaust noise respectively. Table 2a gives the locations and angles of each microphone in the inlet focused array and Table $2 \mathrm{~b}$ gives the locations and angles of each microphone in the exhaust focused array. The microphones are positioned at the height (z-axis) of the engine centerline.

\section{II.B.2. 88-Inch Sideline Array (88SL)}

As a mobile test rig, there is the potential to use the DART in the NASA GRC 9x15 LSWT. This wind tunnel uses a traversing microphone system to create a virtual sideline microphone array approximately 88-inches from the model centerline. Therefore, an 88-inch sideline array was deployed for the DART baseline test. Microphone locations and polar angles for the 88SL array are given in Table 3.

\section{II.B.3. Overhead Array (24FF)}

The overhead $(24 \mathrm{FF})$ array is fixed to the AAPL dome structure. The $24 \mathrm{FF}$ array is an arc array with a 50 -foot radius centered on the NATR near the center of the dome. Therefore, since the DGEN380 is not at the center of the arc, microphone distance, polar, and azimuthal angles vary across the array. Table 4 shows the coordinate locations relative to the DGEN380 center (Table 1) and the corresponding radial distance, 
Table 2: 10-foot arc array microphone locations in the DGEN coordinate system (Figure 2).

\begin{tabular}{|c|c|c|c|c|}
\hline Mic & Polar Angle & $\mathrm{x}(\mathrm{ft})$ & $\mathrm{y}(\mathrm{ft})$ & $\mathrm{z}(\mathrm{ft})$ \\
\hline 1 & 0 & 10.00 & 0.00 & 0 \\
\hline 2 & 6.4 & 9.94 & 1.11 & 0 \\
\hline 3 & 12.9 & 9.75 & 2.23 & 0 \\
\hline 4 & 19.3 & 9.44 & 3.31 & 0 \\
\hline 5 & 25.7 & 9.01 & 4.34 & 0 \\
\hline 6 & 32.1 & 8.47 & 5.31 & 0 \\
\hline 7 & 38.6 & 7.82 & 6.24 & 0 \\
\hline 8 & 45 & 7.07 & 7.07 & 0 \\
\hline 9 & 51.4 & 6.24 & 7.82 & 0 \\
\hline 10 & 57.9 & 5.31 & 8.47 & 0 \\
\hline 11 & 64.3 & 4.34 & 9.01 & 0 \\
\hline 12 & 70.7 & 3.31 & 9.44 & 0 \\
\hline 13 & 77.1 & 2.23 & 9.75 & 0 \\
\hline 14 & 83.6 & 1.11 & 9.94 & 0 \\
\hline 15 & 90 & 0.00 & 10.00 & 0 \\
\hline
\end{tabular}

(a) Inlet-Centered Array

\begin{tabular}{|c|c|c|c|c|}
\hline Mic & Polar Angle & $\mathrm{x}(\mathrm{ft})$ & $\mathrm{y}(\mathrm{ft})$ & $\mathrm{z}(\mathrm{ft})$ \\
\hline 16 & 90 & 0.00 & 10.00 & 0 \\
\hline 17 & 95 & -0.87 & 9.96 & 0 \\
\hline 18 & 100 & -1.74 & 9.85 & 0 \\
\hline 19 & 105 & -2.59 & 9.66 & 0 \\
\hline 20 & 110 & -3.42 & 9.40 & 0 \\
\hline 21 & 115 & -4.23 & 9.06 & 0 \\
\hline 22 & 120 & -5.00 & 8.66 & 0 \\
\hline 23 & 125 & -5.74 & 8.19 & 0 \\
\hline 24 & 130 & -6.43 & 7.66 & 0 \\
\hline 25 & 135 & -7.07 & 7.07 & 0 \\
\hline 26 & 140 & -7.66 & 6.43 & 0 \\
\hline 27 & 145 & -8.19 & 5.74 & 0 \\
\hline 28 & 150 & -8.66 & 5.00 & 0 \\
\hline 29 & 155 & -9.06 & 4.23 & 0 \\
\hline 30 & 160 & -9.40 & 3.42 & 0 \\
\hline
\end{tabular}

(b) Exhaust-Centered Array 
Table 3: Microphone locations relative to the center of the DGEN for the 88-inch sideline array in the DGEN coordinate system (Figure 2).

\begin{tabular}{|c|c|c|c|c|}
\hline Channel & $\theta$ & $\mathrm{x}(\mathrm{ft})$ & $y(\mathrm{ft})$ & $\mathrm{z}(\mathrm{ft})$ \\
\hline 1 & 15 & 7.083 & 1.898 & 0 \\
\hline 2 & 20 & 6.891 & 2.508 & 0 \\
\hline 3 & 25 & 6.646 & 3.099 & 0 \\
\hline 4 & 30 & 6.351 & 3.667 & 0 \\
\hline 5 & 35 & 6.007 & 4.206 & 0 \\
\hline 6 & 40 & 5.618 & 4.714 & 0 \\
\hline 7 & 45 & 5.185 & 5.185 & 0 \\
\hline 8 & 50 & 4.714 & 5.618 & 0 \\
\hline 9 & 55 & 4.206 & 6.007 & 0 \\
\hline 10 & 60 & 3.667 & 6.351 & 0 \\
\hline 11 & 65 & 3.099 & 6.646 & 0 \\
\hline 12 & 70 & 2.508 & 6.891 & 0 \\
\hline 13 & 75 & 1.898 & 7.083 & 0 \\
\hline 14 & 80 & 1.273 & 7.222 & 0 \\
\hline 15 & 85 & 0.639 & 7.305 & 0 \\
\hline 16 & 90 & 0.000 & 7.333 & 0 \\
\hline 17 & 95 & -0.639 & 7.305 & 0 \\
\hline 18 & 100 & -1.273 & 7.222 & 0 \\
\hline 19 & 105 & -1.898 & 7.083 & 0 \\
\hline 20 & 110 & -2.508 & 6.891 & 0 \\
\hline 21 & 115 & -3.099 & 6.646 & 0 \\
\hline 22 & 120 & -3.667 & 6.351 & 0 \\
\hline 23 & 125 & -4.206 & 6.007 & 0 \\
\hline 24 & 130 & -4.714 & 5.618 & 0 \\
\hline 25 & 135 & -5.185 & 5.185 & 0 \\
\hline 26 & 140 & -5.618 & 4.714 & 0 \\
\hline 27 & 145 & -6.007 & 4.206 & 0 \\
\hline 28 & 150 & -6.351 & 3.667 & 0 \\
\hline 29 & 155 & -6.646 & 3.099 & 0 \\
\hline 30 & 160 & -6.891 & 2.508 & 0 \\
\hline
\end{tabular}




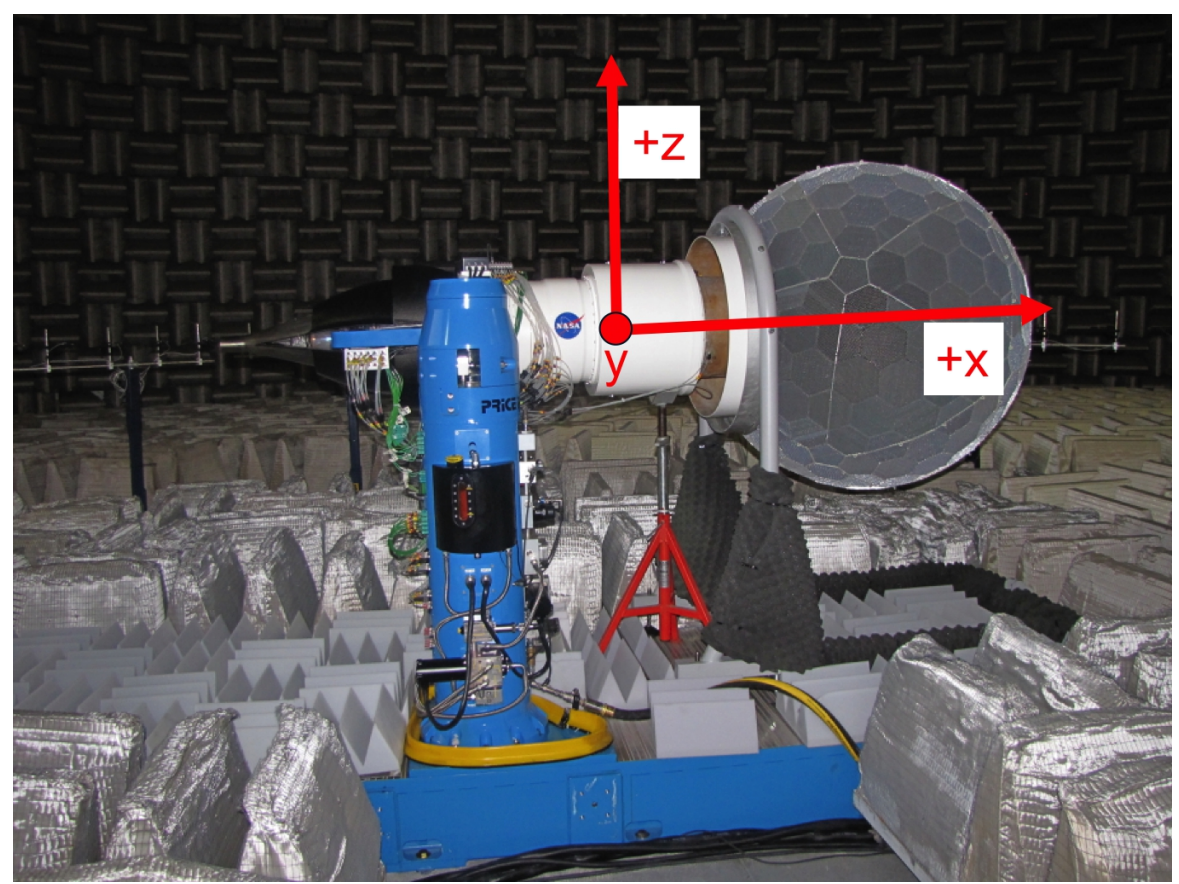

Figure 2: DGEN380 viewed from the pylon side looking toward the microphone arrays. The lines indicate the microphone coordinate system with the positive y-axis into the page. Origin coordinates for the different source locations are given in Table 1.

polar $(\theta)$ angle, and azimuthal $(\phi)$ angle. The $24 \mathrm{FF}$ array was fully independent of the other arrays; data were acquired using $24 \mathrm{FF}$ for every run along with the $10 \mathrm{FT}$ or $88 \mathrm{SL}$ data.

\section{II.C. Data Acquisition and Processing}

Performance and acoustic data were acquired using separate systems. The engine performance data, along with the ambient atmospheric conditions, were recorded by the engine control system and output as a tabular flat database file. These data were recorded at $1 \mathrm{~Hz}$ sample rate and averaged over the time of each acoustic data point to get one value for each field. A flag in the engine data system is set when the engine is on condition and the engine control computer sends a TTL pulse to the acoustic data systems to start data acquisition.

Two separate acoustic data acquisition systems were used for the DART baseline test. The first, which will be the standard DART acoustic data acquisition system, was used for the 10FT and 88SL arrays. The second is the standard acquisition system for the AAPL 24FF array. Each system will be described below.

\section{II.C.1. Arc and Sideline Arrays (10FT and 88SL)}

The 10FT and 88SL arrays used 1/4" Electret-style PCB model 378C01 microphones with integrated preamplifiers. These microphones are TEDS capable current-supplied at $6 \mathrm{~mA}$. Acoustic data from these arrays were acquired using a Genesis GEN2i 24-bit data recorder from HBM. Data were digitized using a 24-bit analog-to-digital converter at a $250 \mathrm{kHz}$ sample rate. A $50 \mathrm{~Hz}$ to $50 \mathrm{kHz}$ bandwidth filter was applied to remove the DC offset and any very low frequency background noise while also serving as an anti-aliasing Nyquist filter. Once-per-revolution signals from the high pressure turbine (HPT) and low pressure turbine (LPT) were also recorded to track the exact engine speed. This system is installed in the DART control truck and, therefore, travels with the DART as the standard data acquisition system.

\section{II.C.2. Overhead Array (24FF)}

The 24FF array uses 24 Bruel \& Kjær Type 3949 1/4" microphones. Bruel \& Kjær Nexus units provide amplification and signal conditioning. Data is digitized and recorded on a DataMAX Instrumentation 
Table 4: AAPL overhead array $(24 \mathrm{FF})$ microphone locations relative to the center of the DGEN in the DGEN coordinate system (Figure 2).

\begin{tabular}{|c|c|c|c|c|c|c|}
\hline Channel & $\mathrm{x}(\mathrm{ft})$ & $\mathrm{y}(\mathrm{ft})$ & $\mathrm{z}(\mathrm{ft})$ & $\mathrm{R}(\mathrm{ft})$ & $\theta(\mathrm{deg})$ & $\phi(\mathrm{deg})$ \\
\hline 1 & 38.5 & 32.5 & -17.0 & 53.1 & 43.6 & -27.6 \\
\hline 2 & 37.4 & 35.6 & -14.9 & 53.7 & 45.9 & -22.7 \\
\hline 3 & 34.2 & 37.0 & -12.9 & 52.0 & 48.9 & -19.3 \\
\hline 4 & 31.1 & 39.1 & -10.7 & 51.0 & 52.5 & -15.3 \\
\hline 5 & 28.4 & 40.4 & -8.8 & 50.1 & 55.5 & -12.4 \\
\hline 6 & 25.2 & 41.5 & -6.7 & 49.0 & 59.1 & -9.1 \\
\hline 7 & 21.7 & 42.6 & -5.1 & 48.1 & 63.2 & -6.8 \\
\hline 8 & 18.4 & 43.5 & -3.4 & 47.4 & 67.1 & -4.5 \\
\hline 9 & 14.3 & 44.0 & -2.0 & 46.3 & 72.0 & -2.7 \\
\hline 10 & 11.0 & 44.0 & -0.6 & 45.4 & 76.0 & -0.7 \\
\hline 11 & 7.3 & 43.7 & 0.7 & 44.4 & 80.5 & 0.9 \\
\hline 12 & 3.3 & 43.7 & 1.5 & 43.8 & 85.7 & 1.9 \\
\hline 13 & -0.3 & 42.7 & 2.4 & 42.8 & 90.4 & 3.2 \\
\hline 14 & -4.6 & 41.5 & 2.9 & 41.9 & 96.3 & 4.0 \\
\hline 15 & -7.8 & 40.7 & 3.5 & 41.6 & 100.8 & 4.8 \\
\hline 16 & -11.3 & 39.1 & 3.8 & 40.9 & 106.1 & 5.6 \\
\hline 17 & -14.9 & 37.3 & 4.0 & 40.3 & 111.7 & 6.1 \\
\hline 18 & -18.1 & 35.2 & 3.7 & 39.8 & 117.1 & 6.1 \\
\hline 19 & -21.3 & 33.0 & 3.5 & 39.4 & 122.7 & 6.1 \\
\hline 20 & -24.3 & 30.2 & 3.1 & 38.9 & 128.7 & 5.9 \\
\hline 21 & -27.1 & 27.5 & 2.4 & 38.7 & 134.5 & 5.0 \\
\hline 22 & -29.5 & 24.7 & 1.7 & 38.5 & 140.1 & 3.9 \\
\hline 23 & -32.0 & 21.6 & 0.5 & 38.6 & 146.0 & 1.3 \\
\hline 24 & -33.9 & 18.4 & -0.4 & 38.5 & 151.5 & -1.4 \\
\hline & & & & & & \\
\hline
\end{tabular}


Recorder from R.C. Electronics using a 16-bit analog-to-digital converter, a $200 \mathrm{kHz}$ sample rate, and a $90 \mathrm{kHz}$ Nyquist filter.

\section{II.C.3. Data Processing}

Time series acquired on the Genesis and DataMAX recorders are first converted from their native format into a general one-file-per-channel format for processing. These time series are then converted into the frequency domain using a standard fast Fourier transform routine based on $2^{14}$ point Kaiser window with $50 \%$ overlap. Once in the frequency domain, the background noise, acquired before each run, is subtracted; any frequency where the measured engine noise is less than $3 \mathrm{~dB}$ above the background noise is removed from the spectra. Next, the spectra are corrected to account for the free-field response of each individual microphone.

Finally, the corrected spectra are projected to a 1-foot radius arc by accounting for atmospheric losses ${ }^{5}$ and the spherical spreading of sound. This projection assumes that the microphone is in the acoustic field defined as the region outside of the flow where pressure waves propagate at the speed of sound and the spherical spreading assumption is valid. The geometric far-field is a special subset of the acoustic field defined by distance beyond which the engine can be considered a point source for distance scaling and atmospheric corrections. Noise measured in the acoustic field but not the geometric far-field can be projected to the 1-foot lossless condition but a more detailed knowledge of the noise source distribution produced by the engine is required; the projection to the 1-foot lossless condition in this case is discussed in Section IV.

\section{II.D. Engine Operating Points}

The engine operating points are defined by corrected fan speed. Each operating speed will be assigned a setpoint number for easier identification. Table 5 shows the standard matrix of engine operating points along with setpoint number. The engine control unit is programmed to cycle through this matrix remaining at each programmed speed for 30 seconds before proceeding to the next point. The control unit sets a flag in the output file and sends a TTL pulse when on condition to notify external (acoustic) data acquisition systems to acquire data.

Table 5: Standard test matrix of 11 engine operating points with 3 repeats defined by engine speed and assigned setpoint ID numbers. Note than engine speed is a percent of maximum corrected fan RPM. The "Daily Maximum" represents the maximum engine speed for the ambient conditions on each day.

\begin{tabular}{|c|c|c|}
\hline Sequence & Setpoint & Engine Speed \\
\hline 0 & 9900 & Background (0\%) \\
\hline 1 & 1 & Idle \\
\hline 2 & 2 & $50.0 \%$ \\
\hline 3 & 3 & $60.0 \%$ \\
\hline 4 & 4 & $70.0 \%$ \\
\hline 5 & 5 & $80.0 \%$ \\
\hline 6 & 6 & $90.0 \%$ \\
\hline 7 & 7 & $92.5 \%$ \\
\hline 8 & 8 & Daily Maximum \\
\hline 9 & 2 & $50.0 \%$ \\
\hline 10 & 8 & Daily Maximum \\
\hline 11 & 1 & Idle \\
\hline
\end{tabular}

\section{Analysis of Baseline Data}

The noise characteristics of the DGEN380 were initially measured in 2014 using a truck-mounted demonstration engine. ${ }^{1}$ These measurements are repeated in the current test to create a noise baseline for the production engine in a research configuration. The DGEN380 creates a noise spectra consisting of a broadband base sprinkled with tones produced by the various rotating components (Figure 3). The source of these 
tones, labeled in Figure 3, can be identified by frequency using the engine speed and the number of blades on the component. At broadside angles, the $24 \mathrm{FF}$ array shows spectral features from both the inlet and exhaust as shown by the presence of fan, compressor, low-pressure turbine (LPT), and high-pressure turbine (HPT) blade passing tones in Figure 3. The second (F2) and third (F3) harmonics of the fan BPF tone have the highest amplitude in the audible range, approximately $15 \mathrm{~dB}$ above the broadband level and $6 \mathrm{~dB}$ above the fan fundamental BPF tone. The compressor BPF tone $(\mathrm{C} 1)$ and first harmonic $(\mathrm{C} 2)$ are more than 6 $\mathrm{dB}$ below the peak fan tones. The first interaction tone corresponds to the $\mathrm{F} 1+\mathrm{C} 1$ frequency and has an amplitude slightly greater than the $\mathrm{C} 1$ tone.

The highest amplitude tone in the spectra corresponds to the LPT BPF (LPT1). Both the LPT1 and the HPT BPF tone (HPT1) are haystacked which is likely caused by the spreading of the acoustic energy into neighboring frequency bins as the tone is slightly decorrelated traveling through the jet shear-layer to reach the microphone. Note that the LPT1 and HPT1 frequencies are above the audible range and, therefore, do not contribute to the effective perceived noise level (EPNL). However, data acquired using the DART may be scaled to make noise predictions for other applications and, therefore, it is important to document these tones.

Figure 4 shows spectra measured using the $24 \mathrm{FF}$ array at $\theta \approx 45^{\circ}, \theta \approx 90^{\circ}$ and $\theta \approx 145^{\circ}$ with the DGEN operating at $90 \%$ speed. At the upstream $\theta \approx 45^{\circ}$ location, the several fan and compressor tones appear in pairs starting around $9 \mathrm{kHz}$ (the $\mathrm{C} 1$ frequency) and the broadband noise is steadily declining as frequency increases. Around $\theta \approx 90^{\circ}$ the fan tones are still present but the compressor tones are significantly reduced. The broadband noise also increases at higher frequencies creating two spectral humps centered at the LPT1 and HPT1 tones; this is the spectral spreading as these tones are more influenced by the flow and jet shear-layer turbulence. Finally, at $\theta \approx 145^{\circ}$ the spectral spreading at the LPT1 and HPT1 tones has converted nearly all the acoustic energy out of the tones and into the broadband humps. Also at this observer angle, the fan tones no longer set the peak spectral amplitude but rather the jet noise is the dominant source (appearing $0 \leq f \leq 1000 \mathrm{~Hz}$ in Figure 4c). The spectra in Figure 4 are replotted on a logarithmic frequency scale in Figure $5 c$ to show the jet noise source at low frequencies and downstream angle.

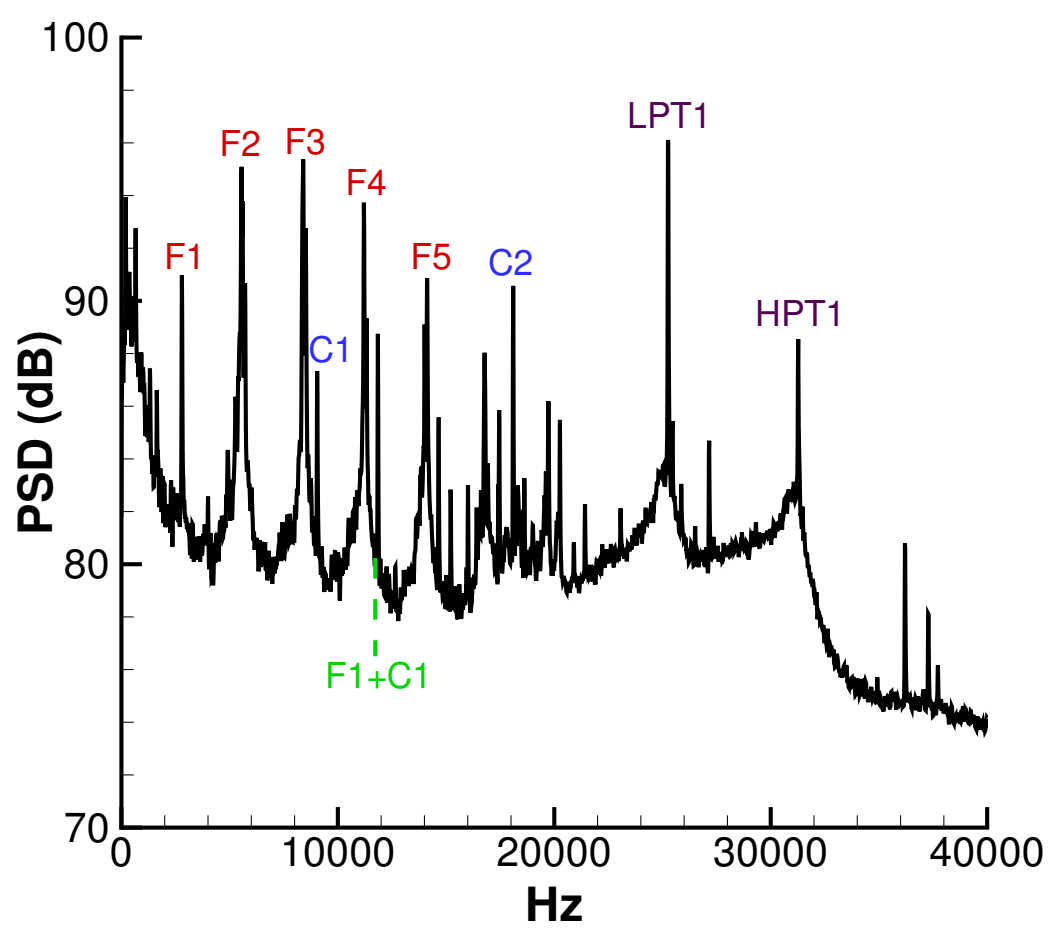

Figure 3: Spectra measured by the $24 \mathrm{FF}$ array at $\theta \approx 90^{\circ}$ to the DGEN, operating at $90 \%$ speed,with labels indicating the source of prominent tones. 


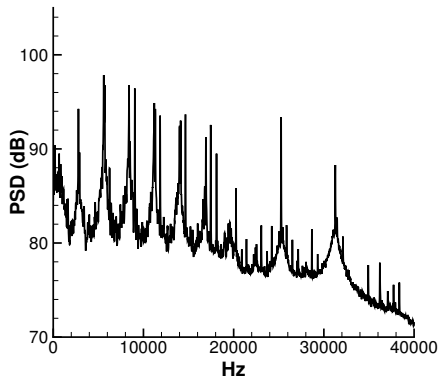

(a) $\theta=45^{\circ}$

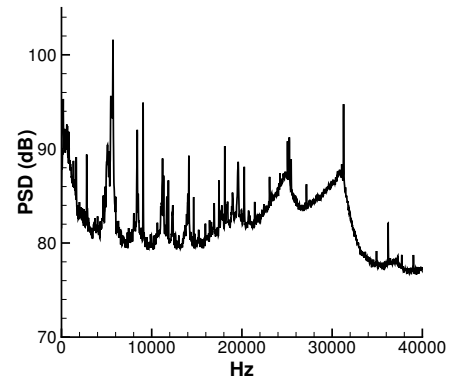

(b) $\theta=90^{\circ}$

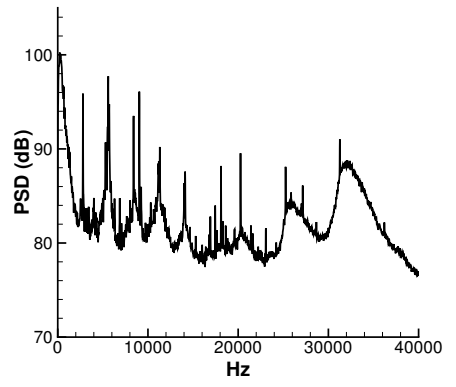

(c) $\theta=145^{\circ}$

Figure 4: Spectra measured by the $24 \mathrm{FF}$ array at $\theta \approx 45^{\circ}, \theta \approx 90^{\circ}$ and $\theta \approx 145^{\circ}$ to the DGEN, operating at $90 \%$.

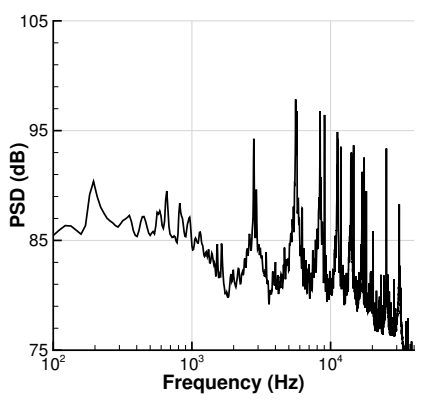

(a) $\theta=45^{\circ}$

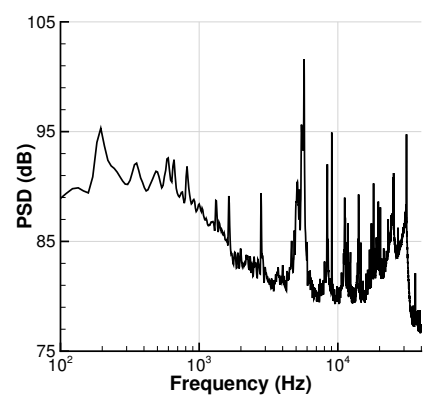

(b) $\theta=90^{\circ}$

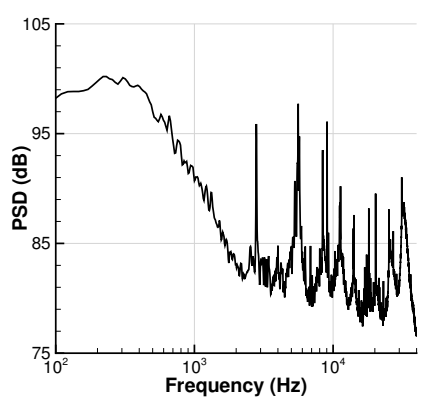

(c) $\theta=145^{\circ}$

Figure 5: Spectra measured by the $24 \mathrm{FF}$ array at $\theta \approx 45^{\circ}, \theta \approx 90^{\circ}$ and $\theta \approx 145^{\circ}$ to the DGEN, operating at $90 \%$. Note the logarithmic frequency scale to show the jet noise spectra. 


\section{III.A. Noise Measurement Repeatability}

Data were acquired using the $24 \mathrm{FF}$ array for all five test runs while the layout of the second array changed (10FT or $88 \mathrm{SL}$ ). Therefore, the $24 \mathrm{FF}$ data can be used to estimate the acoustic measurement repeatability of the DGEN380 across several days with small variations in ambient conditions. Figure 6 shows Overall Sound Pressure Level (OASPL) directivity for the total spectra, the broadband only, and the tones only measured at the $24 \mathrm{FF}$ array $(E S=90 \%$ ) for every run (tone and broadband spectra were separated using the method in [6]). These data show that on an OASPL basis, the measurement repeatability is generally within $\pm 0.5 \mathrm{~dB}$ (there is only one outlier that is only within $\pm 1 \mathrm{~dB}$ of the others at upstream angles).

OASPL is a reasonable metric to estimate the repeatability of broadband noise, and to some extent, the total noise. However, integrated metrics like OASPL can mask differences present in tones which inherently do not repeat as well and can greatly effect frequency-weighted metrics like Effective Perceived Noise Level (EPNL). Figure 7 shows tone amplitude at upstream, broadside, and downstream angles corresponding to the fan, compressor, low-pressure turbine (LPT) and high-pressure turbine (HPT) at $90 \%$ engine speed $(E S=90 \%)$. Individually, these tones repeat within a $\pm 3 \mathrm{~dB}$ which is consistent with previous experience measuring fan tones in wind tunnels. Note that the second harmonic of the compressor tone is an exception to this range, varying by more than $\pm 5 \mathrm{~dB}$. The compressor has 11 full-blades and 11 half-blades so the second harmonic also corresponds to the fundamental frequency when half-blades are included. It is unclear at this point how the half-blades effect the repeatability. Therefore, the DGEN at $E S=90 \%$ gives two repeatability values: $\pm 0.5 \mathrm{~dB}$ for integrated (unweighted) metrics and $\pm 5 \mathrm{~dB}$ for individual tones.

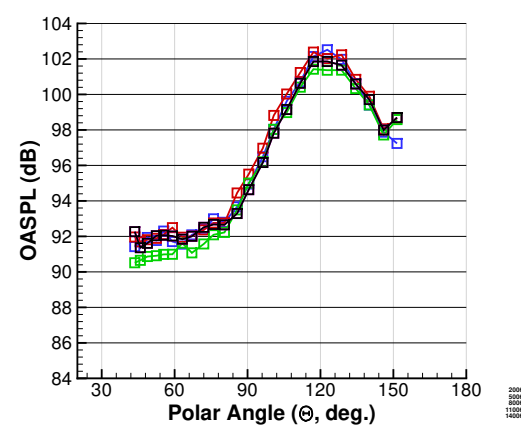

(a) Total

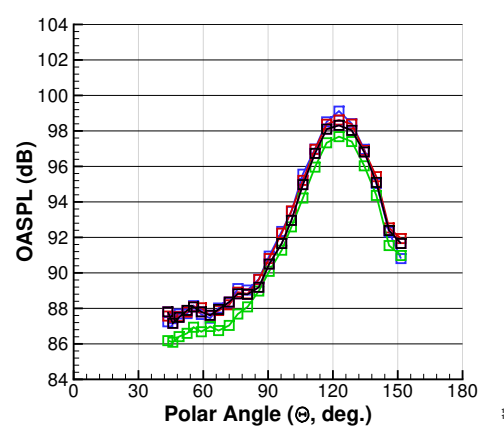

(b) Broadband

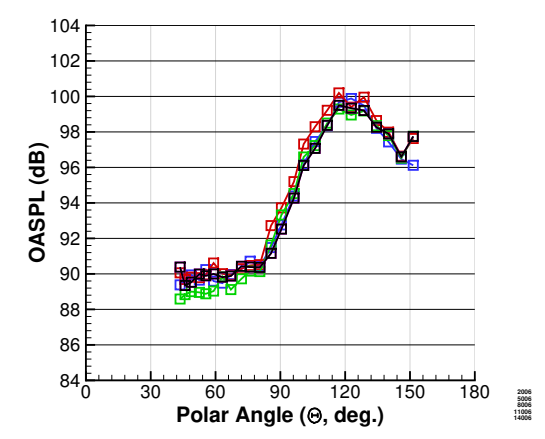

(c) Tone

Figure 6: OASPL $(100 \mathrm{~Hz} \leq f \leq 40 \mathrm{kHz})$ measured by the $24 \mathrm{FF}$ for each repeat at $90 \%$ RPMc. Broadband and tonal components have been separated from the total spectra using the method in [6].

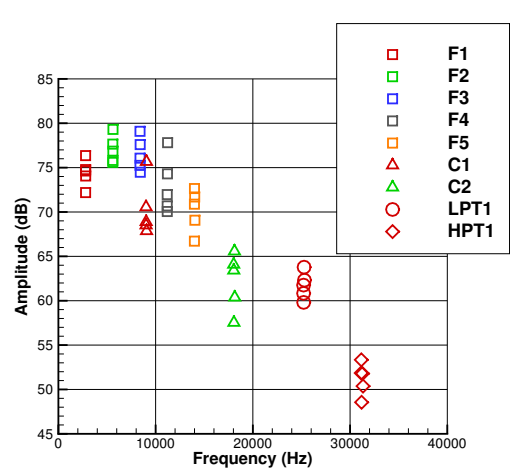

(a) $\theta \approx 45^{\circ}$

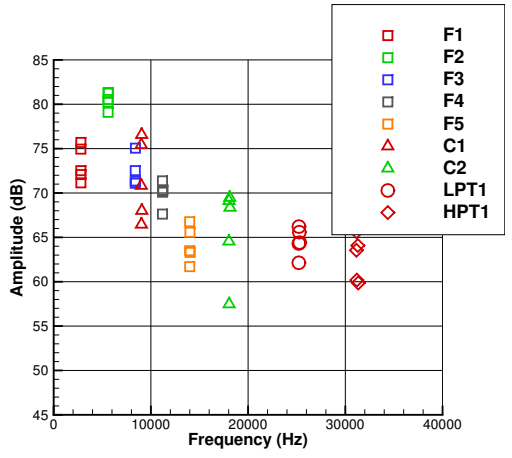

(b) $\theta \approx 90^{\circ}$

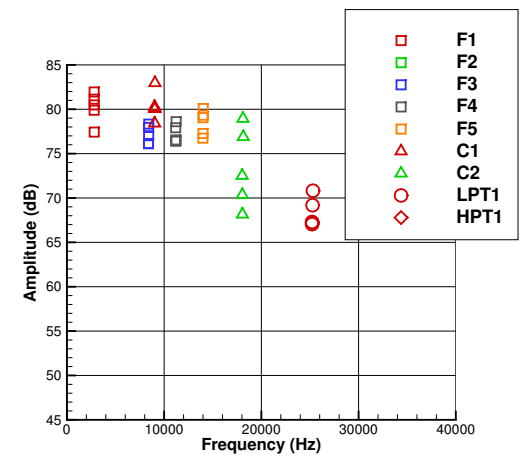

(c) $\theta \approx 135^{\circ}$

Figure 7: Repeatability of tones corresponding to the fan (F1-5), compressor (C1-2), low-pressure turbine (LPT) and high-pressure turbine (HPT) blade passing frequencies acquired by the $24 \mathrm{FF}$ microphone array. 


\section{Projection to 1-Foot Lossless Arc}

The acoustic field of an aircraft engine can be defined as the region outside of the fluctuating hydrodynamic pressure near the inlet, exhaust, and jet plume where the pressure waves travel at the speed of sound for the ambient atmospheric conditions. The acoustic field, therefore, can begin quite close to the engine and extend a significant distance away. The geometric acoustic far-field is a special region in the acoustic field where the observer is sufficiently far away that the engine can be considered at point source for purposes of acoustic propagation. For microphones in the acoustic field but not in the geometric far-field, the location of the inlet or exhaust noise sources is the determining factor that governs the propagation of sound to different distances. Note that the actual distance is not crucial for comparing propagation techniques so a 1-foot-radius arc is used for the presentation of data; this is a commonly used distance for intermediate data processing stages since it is a convenient point for projecting the spectra to other distances.

An aircraft engine is a good example of a system with a distributed noise sources. Tones created by the fan and compressor appear with the associated broadband noise at the fan inlet plane, tone and broadband fan noise appears at the bypass exhaust nozzle, and LPT and HPT tone and broadband noise appear at the core nozzle. The jet exhaust noise from the bypass and core nozzles is a distributed source that extends many nozzle diameters downstream of the engine. Phased-array source localization data acquired on the DGEN engine in 2014 using an Array48 system from OptiNav Inc. ${ }^{7}$ shown in Figure 8, illustrates the distributed nature of the source at several frequencies. Note that the noise field of the DGEN engine is aft dominated with the peak amplitude originating from the fan and core exhaust ducts rather than the inlet. At low frequencies (Figure 8a) the peak source region, defined as the area where the source is within $10 \mathrm{~dB}$ of the maximum, is quite large making it difficult to select just one origin for the sound field. The noise in the higher frequencies originates at the core nozzle exit (Figure 8d) consistent with the previous discussion of HPT and LPT associated noise. Between these two limiting low- and high-frequency ranges, Figures $8 \mathrm{~b}$ and $8 \mathrm{c}$ show competing noise sources at the fan and core exits of similar amplitude with a transition to the dominant core source as the frequency increases; the far-field sound in this frequency range will have contributions from both the fan and core. Note that this analysis is for one engine speed and the actual distribution of sources by frequency will vary as engine speed changes.

The data shown in Figures 9a-9c, acquired at $E S=60 \%$, were processed under the assumption that 10 -foot arc (10FT), 88-inch sideline (88SL) and AAPL overhead (24FF) microphone arrays were in the geometric far-field and, therefore, the center point of the engine accurately represents the noise source origin. At upstream angles (Figure 9a) this assumption appears acceptable through most of the audible range (i.e. $f \leq 20 \mathrm{kHz}$ ) as indicated by the collapse of the spectra onto one (within the repeatability margin discussed in Section III.A). The geometric far-field assumption fails, however, at broadside (Figure 9b) and downstream angles (Figure 9c). Figure 9 shows how the collapse changes if the different possible source orgins (inlet, fan exhaust, or core exhaust) are used to project the spectra to the 1-foot lossless arc and compute the observer angles. For example, the phased-array data does not show any sources within $10 \mathrm{~dB}$ of the peak amplitude near the engine inlet so using the inlet as the origin fails to collapse the data at broadside and downstream angles (Figures 9e-9f). Note that the spectral collapse at the inlet does not change much with different origin choices. Because the noise sources are aft of the engine, the angular difference between the forward observer and the fan or core exit plane is smaller than at broadside or aft angles (in the extreme case where $\theta=0$ there is no directivity change only a small amplitude change caused by slightly different propagation distances). Figure 10 shows similar trends for data acquired at $E S=90 \%$.

When a microphone is in the geometric far-field, the relative angle between these source location and the microphone may be neglected and the point source assumption is valid. Figure 11 shows the case when the microphone is not in the geometric far-field: for microphones at $\theta=90^{\circ}$ relative to the center of the engine, the effective angle to the source (inlet, fan exhaust, core exhaust) changes as the microphone moves farther away. Furthermore, redefining the coordinate system to so that the origin is at one source location (e.g. moving the $\theta=90^{\circ}$ reference to the fan exhaust) will align the sound from that source to a consistent observer angle but increase the angular difference to the other sources. Conversely, as the observer moves toward the geometric far-field, the angle difference between the sources becomes small and distance-scaled spectra begin to collapse. Figures 12 and 13 show spectra measured at the $24 \mathrm{FF}$ array and scaled to the 1-foot lossless condition assuming different source locations on the engine; the near collapse indicates that this array is approaching the geometric far-field. Note that the distance between the DGEN and the 24FF array is considerably less that the typical number of engine lengths that defines the geometric far-field where such a spectra collapse would be expected. It is reiterated the inlet sources are at least $10 \mathrm{~dB}$ below the 
exhaust sources of the critical dimension to define the geometric far-field may be on the order of the distance between the bypass and core nozzles rather than the overall engine length. Another measurement using an array distance based on engine length could confirm this hypothesis.

\section{IV.A. Phased-Array Based Projections}

The analysis thus far has assumed that the sound originates at a single point for all frequencies. However, the phased-array data shows that the sound source location is frequency dependent opening the possibility of using this information to project the spectra using the frequency dependent source location. Figure 14 shows the location of the peak amplitude source for each frequency band in the phased-array data set as a function of axial position for $E S=60 \%$ and $E S=90 \%$. Note that the peak source location moves downstream as the engine speed increases. These peak source locations were used to recompute the spectra by adjusting the measurement location at each frequency, effectively centering the array on the source, before the projection to the one-foot lossless condition. The results are shown in Figures 15 and 16 for $E S=60 \%$ and $E S=90 \%$ respectively. The results are not substantially better than those simply assuming the fan or core exit plane as the origin for two reasons. First, the source region, defined as the area within $10 \mathrm{~dB}$ of the peak, is either large (e.g. Figure 8a) or divided between the fan and core nozzle exits (e.g. Figure 8b) which limits the effectiveness of using only one point as the source. Second, the array spacing in polar angle is too coarse to allow the relatively small changes in directivity angle required for sources that move between the fan and core nozzle exit planes. Note, however, that this is the most basic array processing method and other more advanced methods (e.g. [8,9]may allow a better deconstruction of sources and, therefore, a more accurate projection between arrays.

\section{Summary}

The DART is a fully-mobile engine test rig featuring a DGEN380 geared turbofan based at the NASA Glenn Research Center (GRC) intended for use in aircraft engine noise and performance research. Baseline acoustic and aerodynamic data have been acquired during a test at the NASA GRC AAPL facility using three microphone arrays to investigate the lossless projection of measured noise spectra and to guide future research in other test facilities. These data have also shown that the DART provides good measurement repeatability for broadband noise and repeatability for tone noise consistent with other fan and engine rigs. Analysis of noise data acquired at different distances in the acoustic field demonstrates the need to understand and use the correct noise source origin to project the spectra if the measurement location is not in the geometric far-field. The analysis also indicates the need for future measurements at array distances farther than those possible in the AAPL to confirm the true geometric far-field distance for the DGEN and serve as validation data for source-based data processing methods.

\section{Acknowledgements}

This work was supported by the NASA Advanced Air Vehicle Program, Advanced Air Transport Technology Project. The authors would like to thank Gary Podboy for providing the phased-array data as well as James Bridges and Ed Envia for reviewing this work.

\section{References}

\footnotetext{
${ }^{1}$ Sutliff, D.L., Brown, C.A., Bayon, B., Sree, D., "Farfield Acoustic Characteristics for the DGEN380 Turbofan Engine as Measured in the NASA Glenn Aero-Acoustic Propulsion Laboratory", AIAA 2016-3006, 2016.

${ }^{2}$ Nark, D.M., Jones, M.G., Sutliff, D.L., "Broadband Inlet Liner Design for the DGEN Aero-propulsion Research Turbofan", submitted to 2018 AIAA Aviation Conference.

${ }^{3}$ Bridges, J., "Measurements of Turbulent Flow Field in Separate Flow Nozzles with Enhanced Mixing Devices - Test Report", NASA/TM-2002-211366, 2002.

${ }^{4}$ Sutliff, D., "Acoustic Directivity of the DGEN Aero-propulsion Research Turbofan at Multiple Farfield Array Locations", submitted to the 2018 AIAA Aviation Conference.

5 "Method for Calculation of the Absorption of Sound by the Atmosphere", ANSI S1.26-1995.

${ }^{6}$ Sree, D., "A novel signal processing technique for separating tonal and broadband noise components from counter-rotating open-rotor acoustic data", International Journal of Aeroacoustics, vol. 12, no. 1+2, pp. 169-188, 2013.
} 
${ }^{7}$ Podboy, G., Wernet, M.P., Clem, M.M., Fagan, A.F., "Noise Source Location and Flow Field Measurements on Supersonic Jets and Implications Regarding Broadband Shock Noise", NASA/TM-2017-219544, 2017.

${ }^{8}$ Tester, B.J., Glegg, S.A.L., "A review of engine noise source diagnostic methods for static tests, with phased array and polar correlation techniques", AIAA 2008-2854, 2008.

${ }^{9}$ Tester, B.J., Gabard, G., Glegg, S.A.L., "Spatially distributed models of broadband turbomachinery and jet exhaust noise for engine source breakdown processing of phased array measuements", AIAA 2011-2770, 2011.

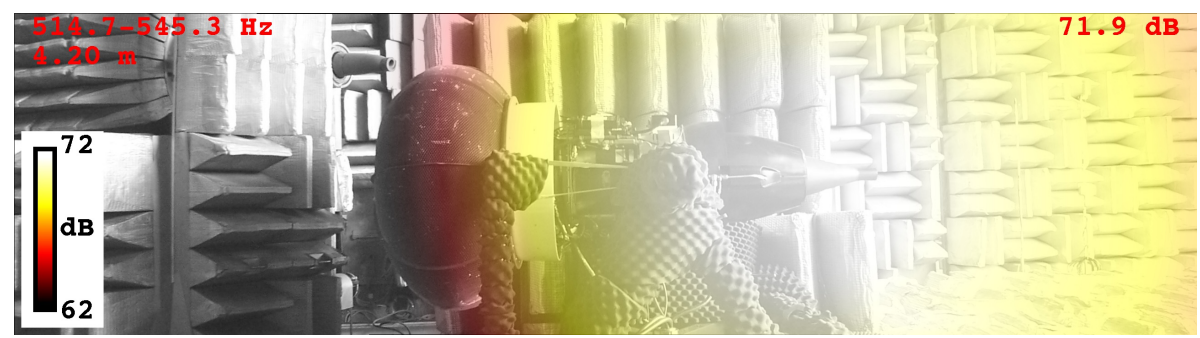

(a) $f=500 \mathrm{~Hz}$

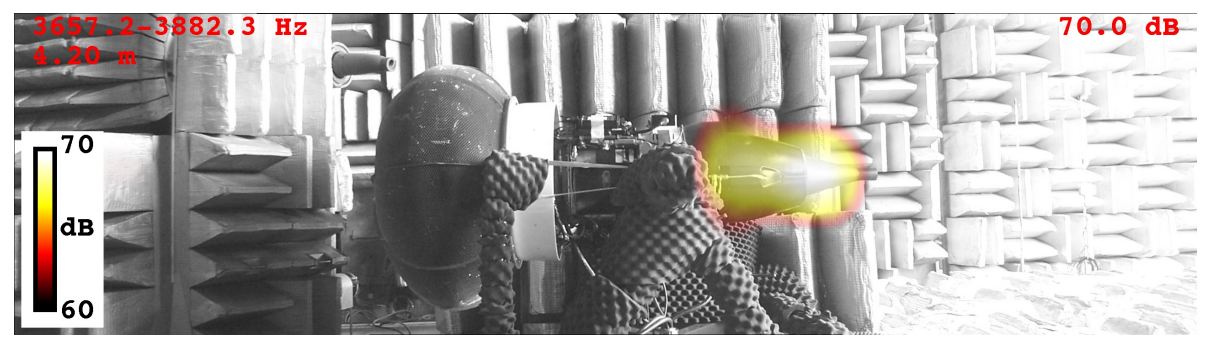

(b) $f=3.7 \mathrm{kHz}$

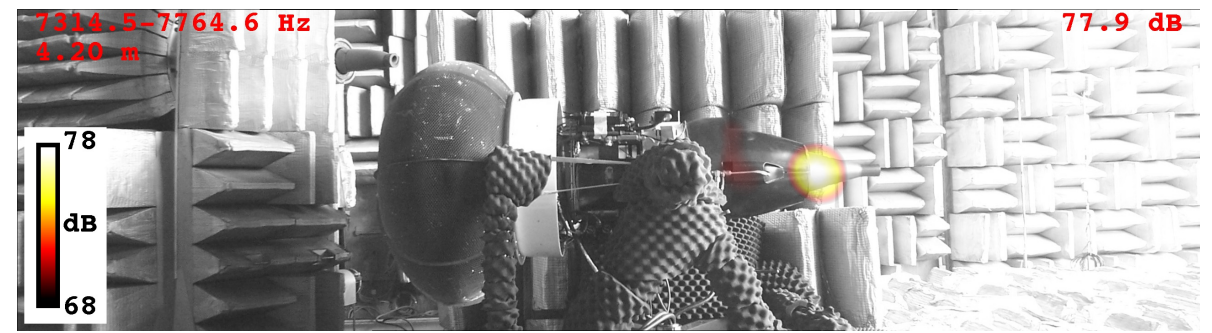

(c) $f=7.5 \mathrm{kHz}$

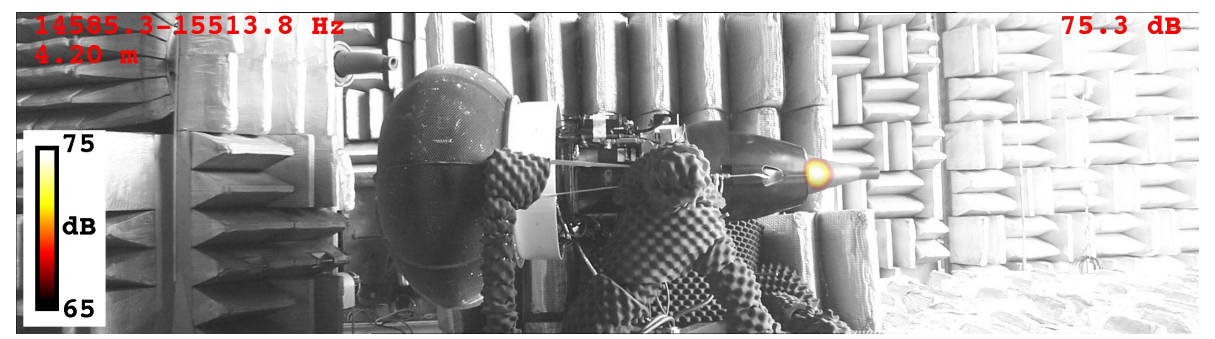

(d) $f=15 \mathrm{kHz}$

Figure 8: Phased-array source localization data at several frequencies acquired at $E S=60 \%$. 


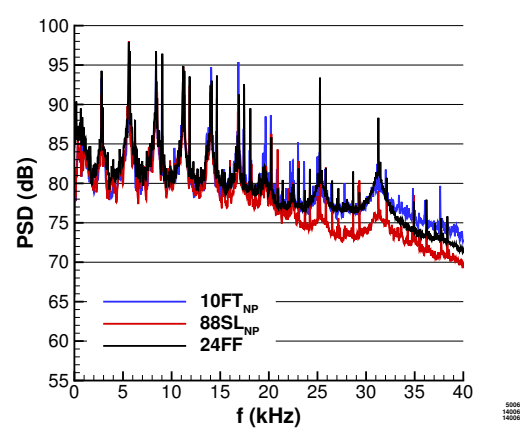

(a) $\theta \approx 45^{\circ}$ re. Engine Center

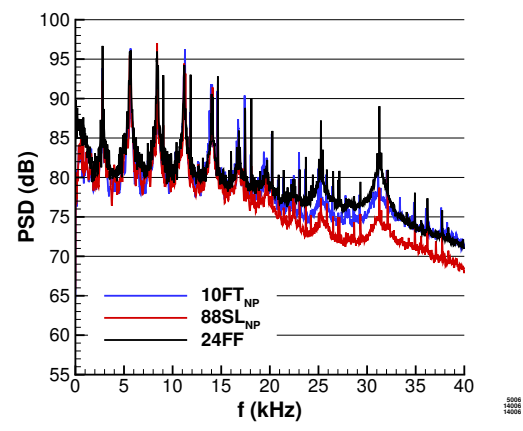

(d) $\theta \approx 45^{\circ}$ re. Inlet

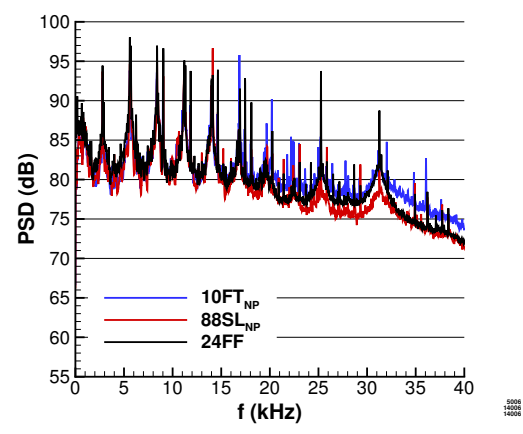

(g) $\theta \approx 45^{\circ}$ re. Fan Exhaust

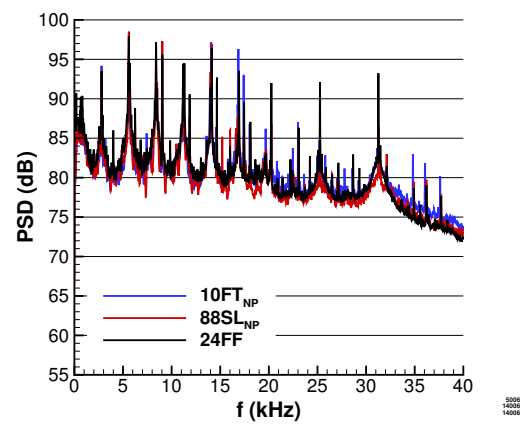

(j) $\theta \approx 45^{\circ}$ re. Core Exhaust

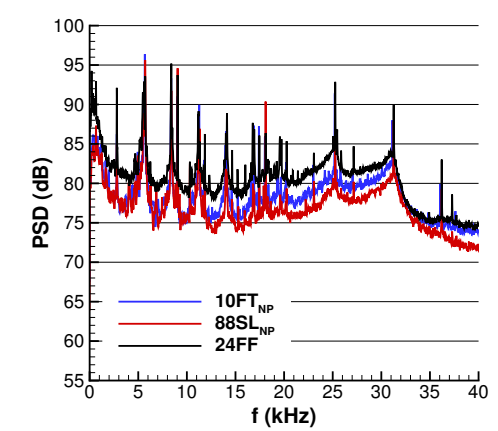

(b) $\theta \approx 80^{\circ}$ re. Engine Center

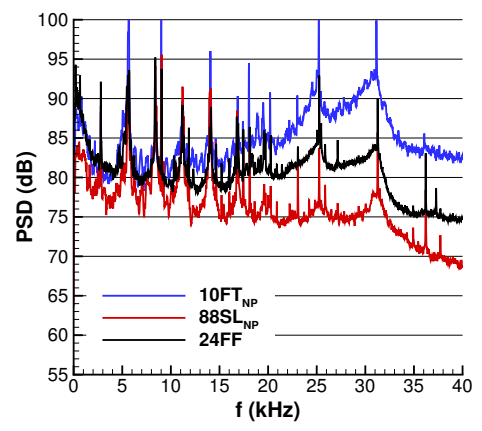

(e) $\theta \approx 80^{\circ}$ re. Inlet

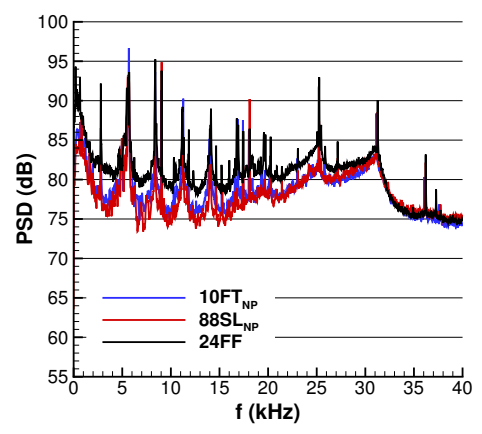

(h) $\theta \approx 80^{\circ}$ re. Fan Exhaust

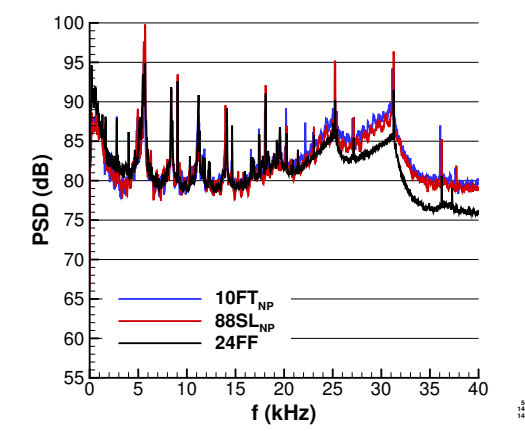

(k) $\theta \approx 80^{\circ}$ re. Core Exhaust

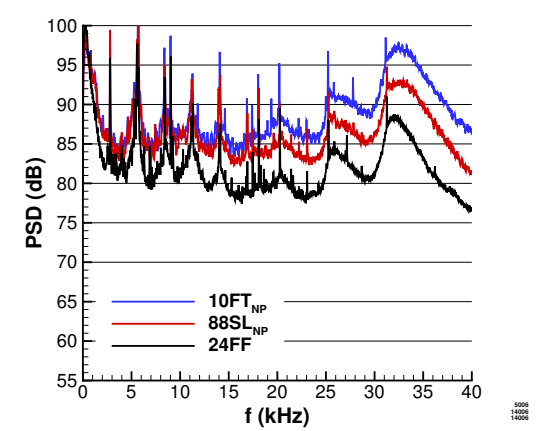

(c) $\theta \approx 145^{\circ}$ re. Engine Center

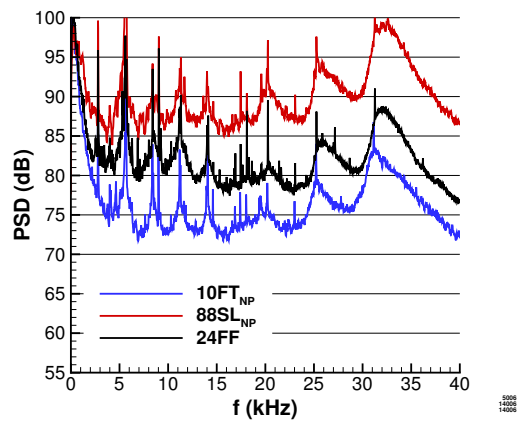

(f) $\theta \approx 145^{\circ}$ re. Inlet

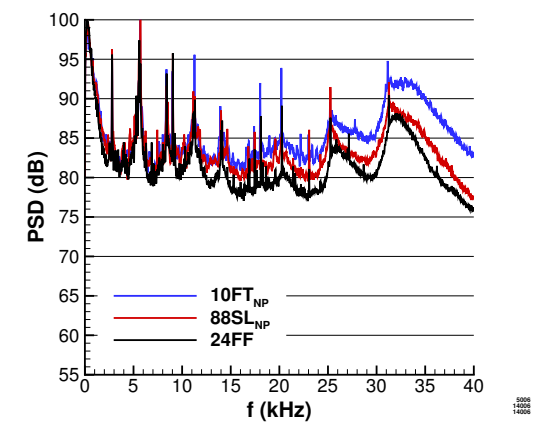

(i) $\theta \approx 145^{\circ}$ re. Fan Exhaust

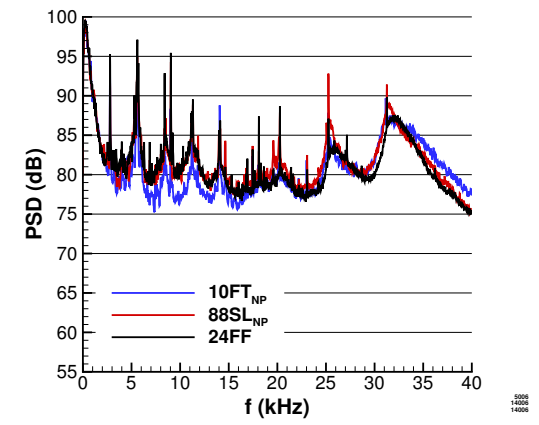

(l) $\theta \approx 145^{\circ}$ re. Core Exhaust

Figure 9: Comparison of spectra measured at the $10 \mathrm{FT}, 88 \mathrm{SL}$, and $24 \mathrm{FF}$ arrays $(E S=60 \%)$ projected to a common 1-foot radius arc accounting for the spherical spreading of sound and atmospheric absorption of sound assuming that all sound originates at the DGEN engine center. 


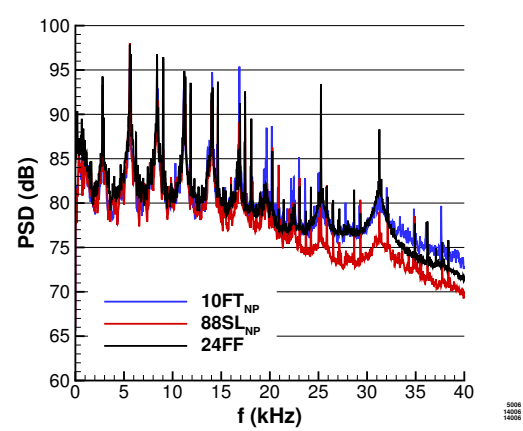

(a) $\theta \approx 45^{\circ}$ re. Engine Center

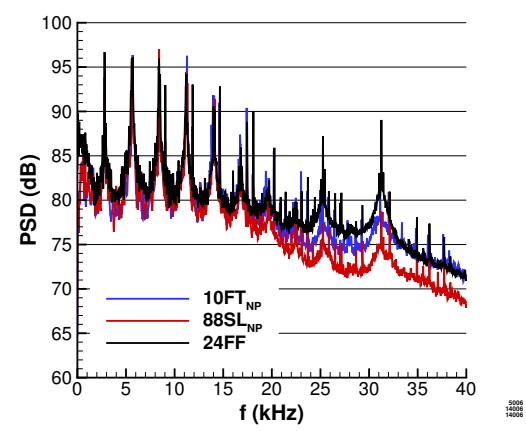

(d) $\theta \approx 45^{\circ}$ re. Inlet

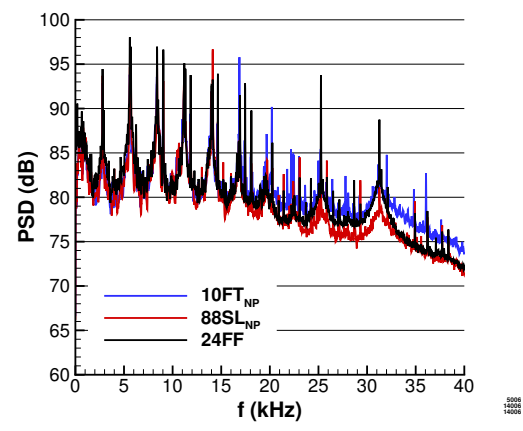

(g) $\theta \approx 45^{\circ}$ re. Fan Exhaust

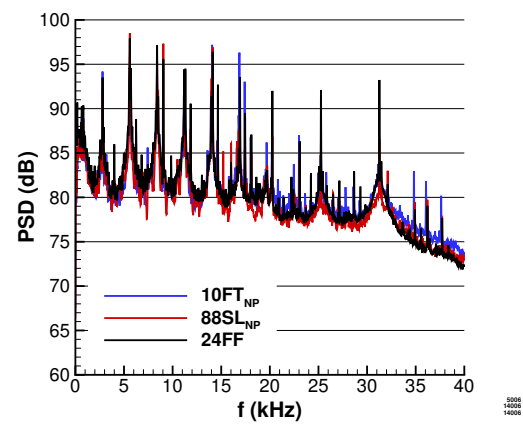

(j) $\theta \approx 45^{\circ}$ re. Core Exhaust

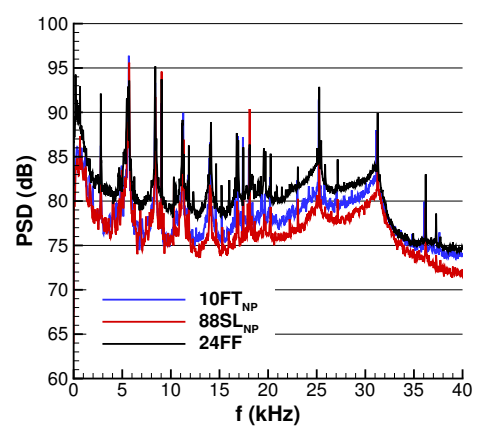

(b) $\theta \approx 80^{\circ}$ re. Engine Center

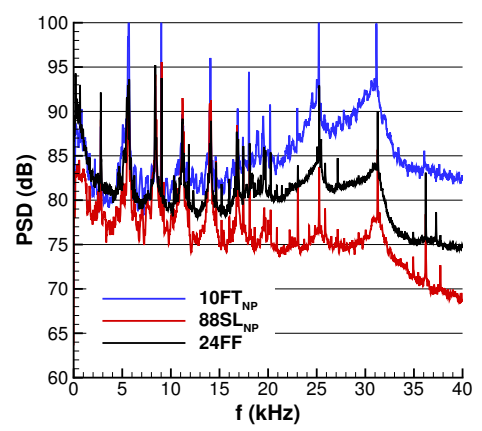

(e) $\theta \approx 80^{\circ}$ re. Inlet

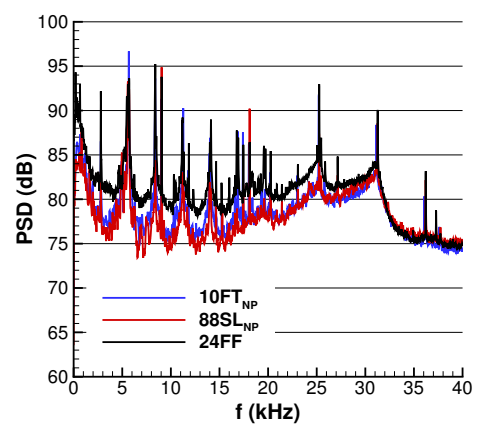

(h) $\theta \approx 80^{\circ}$ re. Fan Exhaust

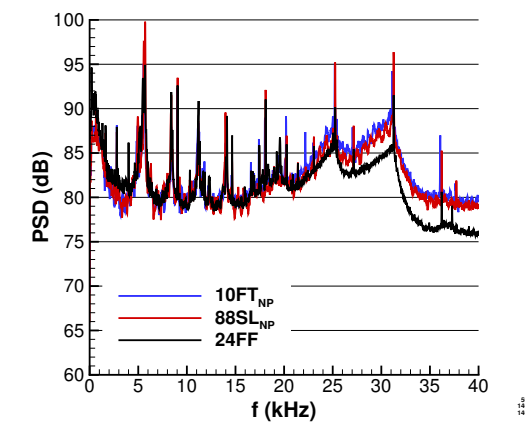

(k) $\theta \approx 80^{\circ}$ re. Core Exhaust

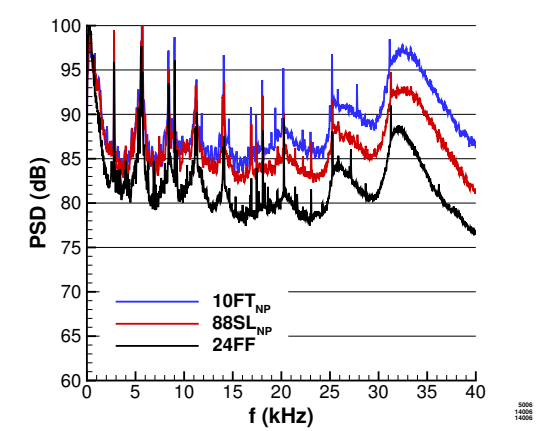

(c) $\theta \approx 145^{\circ}$ re. Engine Center

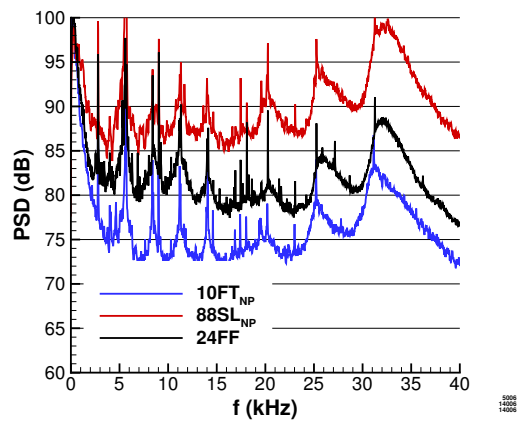

(f) $\theta \approx 145^{\circ}$ re. Inlet

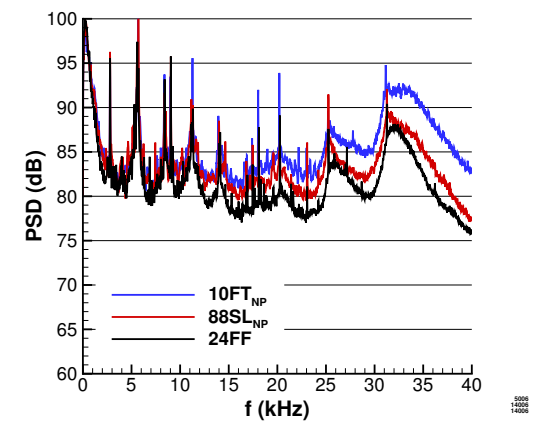

(i) $\theta \approx 145^{\circ}$ re. Fan Exhaust

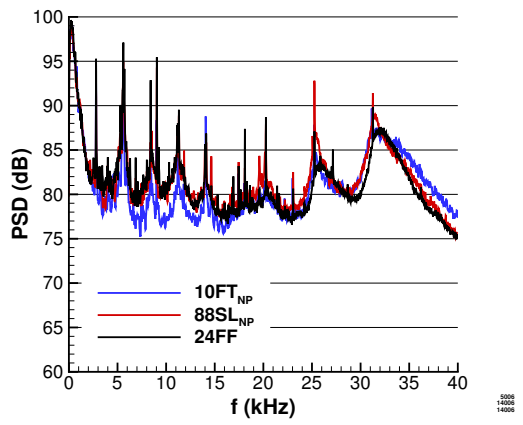

(l) $\theta \approx 145^{\circ}$ re. Core Exhaust

Figure 10: Comparison of spectra measured at the $10 \mathrm{FT}, 88 \mathrm{SL}$, and $24 \mathrm{FF}$ arrays $(E S=90 \%)$ projected to a common 1-foot radius arc accounting for the spherical spreading of sound and atmospheric absorption of sound assuming that all sound originates at the DGEN engine center. 


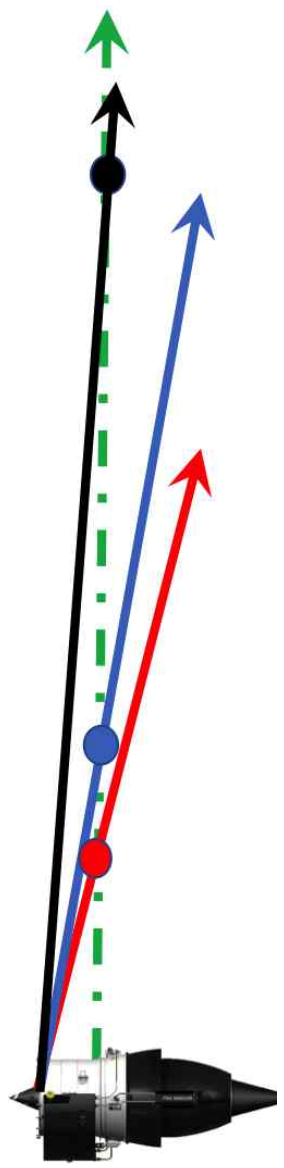

(a) Fan Inlet

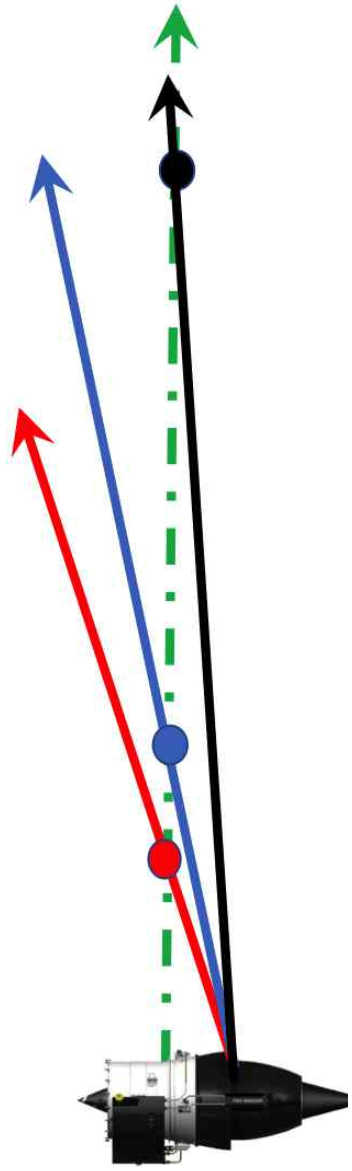

(b) Fan Exhaust

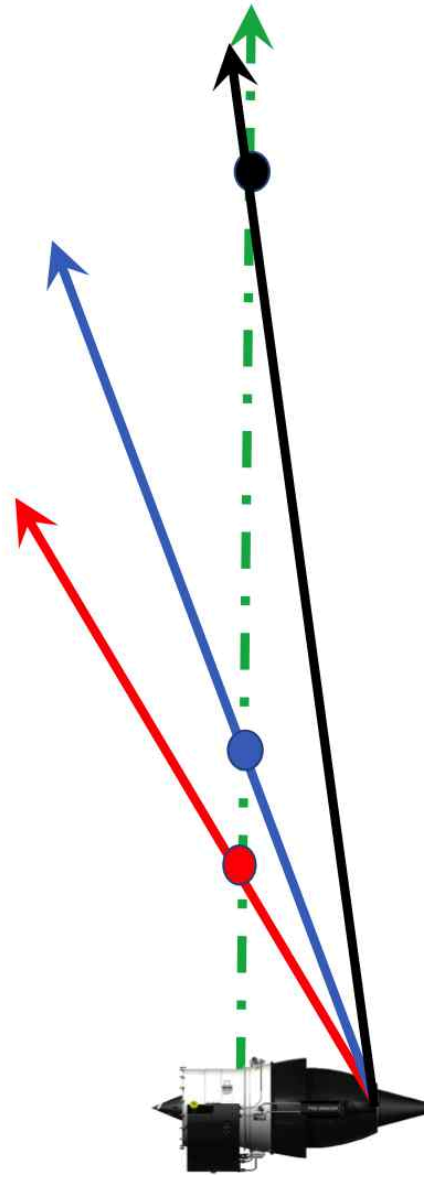

(c) Core Exhaust

Figure 11: Effect of array distance for a microphone at $\theta=90^{\circ}$ to the engine center if the sound source is at the inlet, fan exit plane, or core exit plane. The $88 \mathrm{SL}$ array distance is shown in red, the 10FT is shown in blue, and the $24 \mathrm{FF}$ is shown in black. The dashed green line shows the actual $\theta=90^{\circ}$ line.

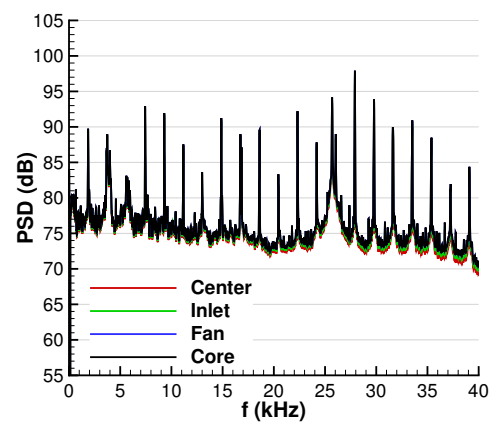

(a) $\theta \approx 45^{\circ}$

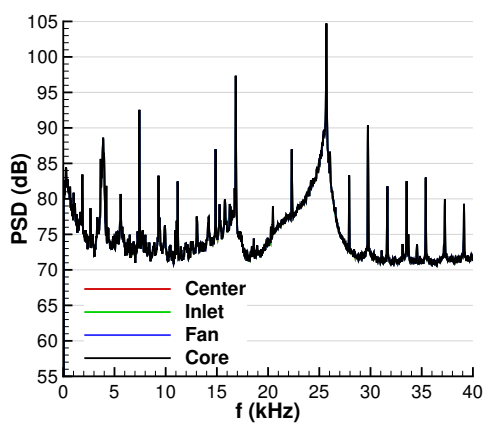

(b) $\theta \approx 90^{\circ}$

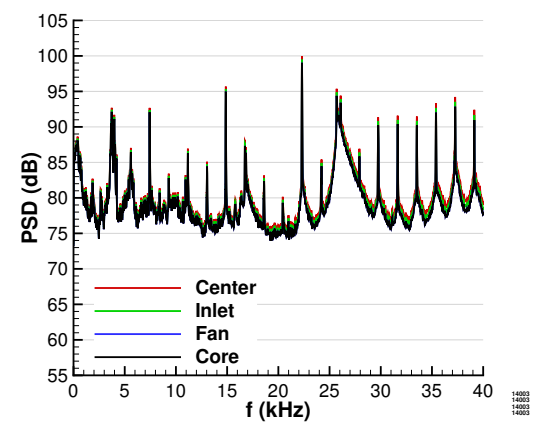

(c) $\theta \approx 145^{\circ}$

Figure 12: Collapse of spectra measured by the $24 \mathrm{FF}$ array at $E S=60 \%$ assuming inlet, engine center, fan exit, and core exit as the noise source origin. 


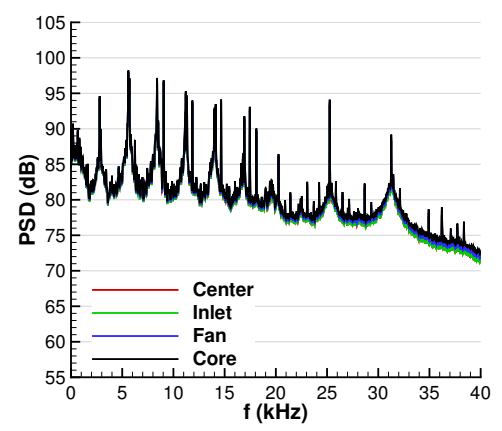

(a) $\theta \approx 45^{\circ}$

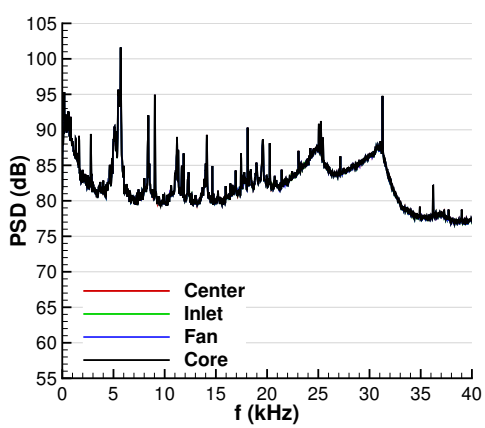

(b) $\theta \approx 90^{\circ}$

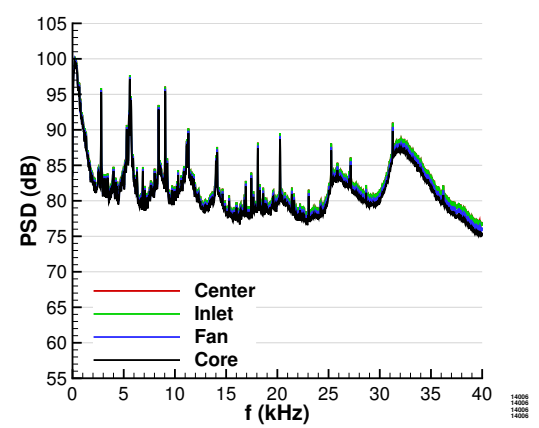

(c) $\theta \approx 145^{\circ}$

Figure 13: Collapse of spectra measured by the $24 \mathrm{FF}$ array at $E S=90 \%$ assuming inlet, engine center, fan exit, and core exit as the noise source origin.

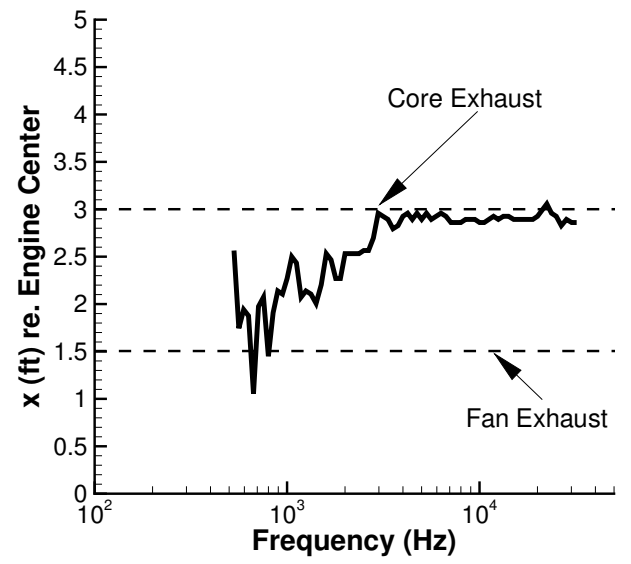

(a) $E S=60 \%$

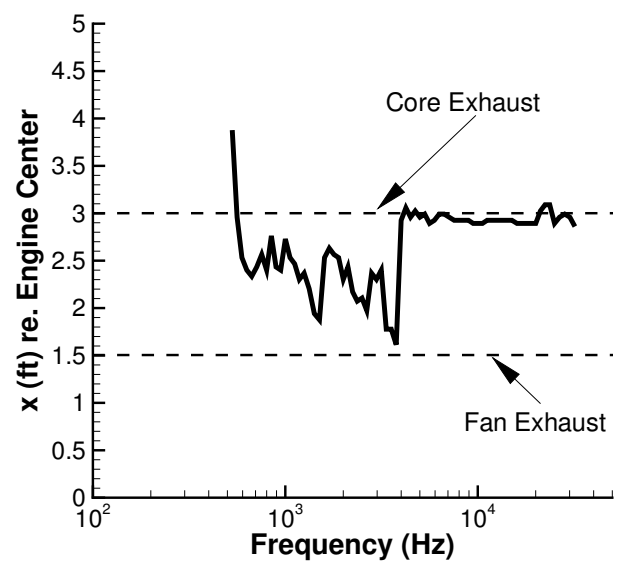

(b) $E S=90 \%$

Figure 14: Axial location, relative to the DGEN center, of the peak source at each frequency measured by the phased-array.

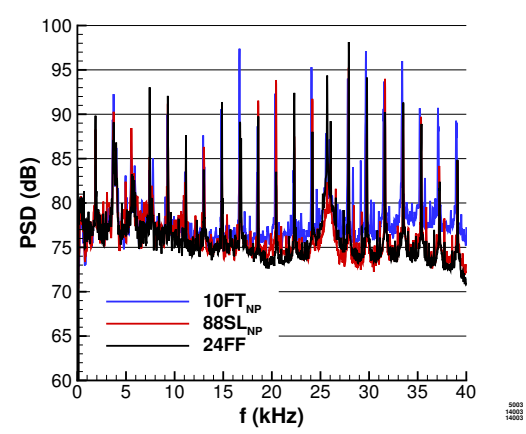

(a) $\theta \approx 45^{\circ}$

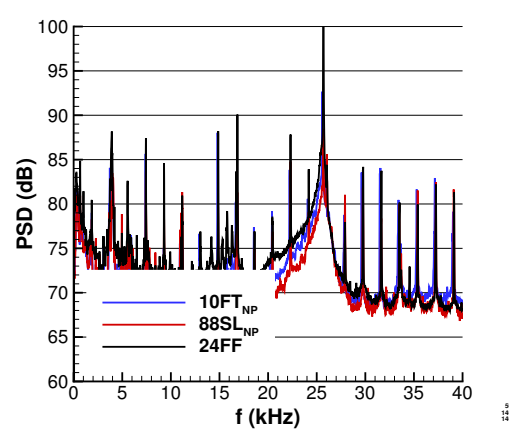

(b) $\theta \approx 80^{\circ}$

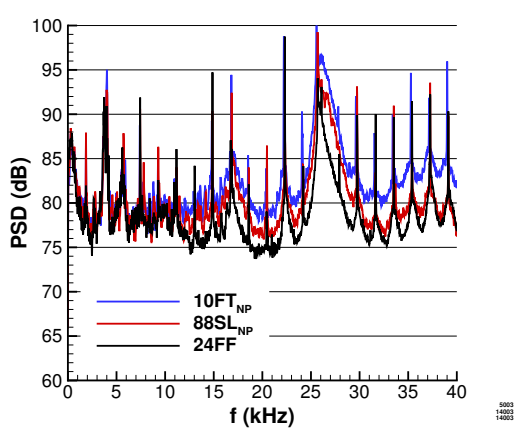

(c) $\theta \approx 145^{\circ}$

Figure 15: Comparison of spectra measured at the $10 \mathrm{FT}, 88 \mathrm{SL}$, and $24 \mathrm{FF}$ arrays $(E S=60 \%)$ projected to a common 1-foot radius arc accounting for the spherical spreading of sound and atmospheric absorption of sound assuming a frequency dependent noise source origin based on the phased-array data in Figure 14a. 


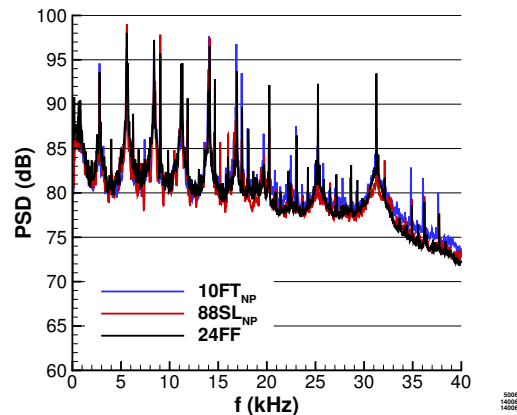

(a) $\theta \approx 45^{\circ}$

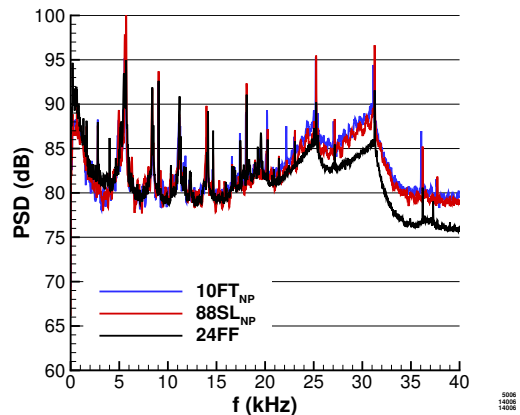

(b) $\theta \approx 80^{\circ}$

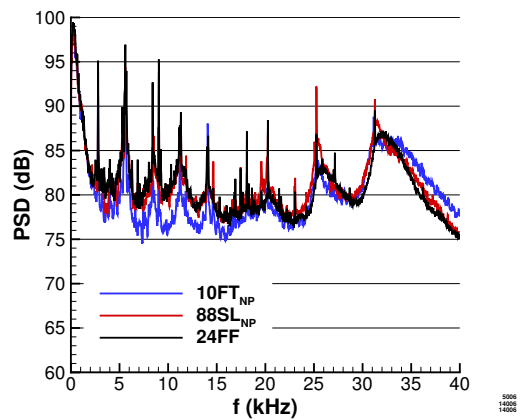

(c) $\theta \approx 145^{\circ}$

Figure 16: Comparison of spectra measured at the $10 \mathrm{FT}, 88 \mathrm{SL}$, and $24 \mathrm{FF}$ arrays $(E S=90 \%)$ projected to a common 1-foot radius arc accounting for the spherical spreading of sound and atmospheric absorption of sound assuming a frequency dependent noise source origin based on the phased-array data in Figure 14b. 